\title{
Sleep disturbance linked to suicidal ideation in postural orthostatic tachycardia syndrome
}

\section{Cathy Lynn Pederson' jill Blettner Brook ${ }^{2}$}

'Department of Biology, Wittenberg University, Springfield, OH, USA;

${ }^{2}$ Patients Count, Anchorage, AK, USA
This article was published in the following Dove Press journal:

Nature and Science of Sleep

12 April 2017

Number of times this article has been viewed

Objective: We investigated the prevalence of suicidal ideation in relationship with symptoms of sleep disruption in people with postural orthostatic tachycardia syndrome (POTS).

Methods: Online surveys (including the Pittsburgh Sleep Quality Index and the Suicide Behaviors Questionnaire - Revised) were completed by 705 POTS patients and 170 non-POTS controls.

Results: Poor sleep quality was reported in $98.4 \%$ of POTS patients with a calculated subjective sleep efficiency of $65.4 \%$. The POTS group's sleep efficiency was significantly lower $(t[873]=-11.32 ; p<0.001)$ and sleep disturbances because of pain were significantly higher $(t[873]=15.36 ; p<0.001)$ than controls. Chi-square testing showed a larger proportion of individuals at high-risk for suicide among POTS patients than controls $\left(\chi^{2}[1, \mathrm{n}=875]=55.6 ; p<0.001\right)$. Multiple linear regression analysis showed that sleep scores $(\beta=0.23, p<0.001)$, age $(\beta=-0.03$, $p<0.001)$, and illness with POTS $(\beta=0.68, p=0.05)$ were significantly associated with suicide ideation scores $(\mathrm{F}[4,870]=38.34, p<0.001)$. This model explained $15 \%$ of variance $\left(R^{2}=0.15\right)$ in suicidal ideation scores.

Conclusion: Patients with POTS may suffer from increased sleep disturbance and suicidal ideation compared with the general population. Treatment to improve sleep efficiency and sleep quality is an important step toward better quality of life for POTS patients.

Keywords: postural orthostatic tachycardia syndrome, POTS, sleep, insomnia, suicidal ideation, suicide

\section{Introduction}

Postural orthostatic tachycardia syndrome (POTS) is a disorder of the autonomic nervous system that manifests with excessive increase in heart rate upon standing (adult $\geq 30$ beats per minute; adolescent $\geq 40$ beats per minute) in the absence of postural hypotension. ${ }^{1}$ Common symptoms include chronic fatigue and pain, as well as diminished quality of life. ${ }^{1-3}$ POTS is a heterogeneous disorder, ${ }^{4}$ and includes symptoms such as neuropathic pain, ${ }^{1}$ poor sleep efficiency, ${ }^{5}$ headaches, palpitations, tremors, gastrointestinal disturbance, facial flushing, ${ }^{6}$ and orthostatic symptoms like dizziness, lightheadedness, and syncope. ${ }^{7}$ Many people have developed POTS after a viral infection, surgery, motor vehicle accident, concussion, or during pregnancy. ${ }^{8,9}$

Many with POTS report sleep disruption at night, ${ }^{6,10-12}$ including poor sleep efficiency and increased sleep fragmentation. ${ }^{5}$ Activation of the sympathetic nervous system may explain sleep disruption in some POTS patients, ${ }^{5,13}$ while sleep disruption could also be due to chronic pain and hyperarousal. ${ }^{11,14}$ In polysomnography studies, people with POTS have a reduction in the heart rate variability for both low frequency
Correspondence: Cathy Pederson Department of Biology, Wittenberg University, PO Box 720, Springfield, $\mathrm{OH} 4550 \mathrm{I}$, USA

Tel + I 937327648 I

Email: cpederson@wittenberg.edu 
and high frequency bands during different sleep stages. ${ }^{11}$ Additionally, POTS patients spend more time in stage two sleep, ${ }^{11}$ and have a reduction in the percentage of rapid eye movement (REM) sleep. ${ }^{15}$

Sleep disruption in POTS and other chronic illnesses should be of clinical concern because insomnia is a known risk factor for suicide. ${ }^{16-18}$ Insomnia has been linked with increased rates of depression, ${ }^{19}$ and approximately $50 \%$ of people with invisible illness suffer from depression, ${ }^{20}$ one of the greatest risk factors for suicide. However, the association between insomnia and suicide remains, even when controlling for depressive disorder ${ }^{21}$ and intensity of depression and hopelessness. ${ }^{22}$ Further, nightmares can be a more potent factor than insomnia in predicting suicide. ${ }^{19,23}$

Suicide is a major cause of death in both the general population and in chronic illness communities where physical and emotional challenges persist. In the general population, suicide is the third leading cause of death in people $<30$ years old. ${ }^{24}$ Increased risk of suicide has been found in chronic fatigue syndrome ${ }^{25,26}$ and fibromyalgia. ${ }^{27-30}$ To our knowledge, no studies of suicide and its relationship to sleep disturbances exist for POTS. Our hypothesis was that increased sleep disturbance in people with POTS would increase the risk of suicidal ideation and suicidal behavior.

\section{Methods}

\section{Participants}

Participants were recruited for the online survey via paid advertising campaigns for people with and without POTS through the Standing Up to POTS Facebook page. From adults over age 18 years old, 705 people with physician diagnosed POTS (688 females, 17 males; mean age 33.75 years; $\mathrm{SD}=11.39$ ) and 170 non-POTS controls ( 145 females, 25 males; mean age 41.90 years; $\mathrm{SD}=14.31$ ) completed the Pittsburgh Sleep Quality Inventory and the Suicide Behavior Questionnaire-Revised. ${ }^{31,32}$ The participants with POTS reported a physician diagnosis of the disorder which was not independently confirmed in this study. They reported being symptomatic for more than a decade $(\bar{x}=11.43$ years, $\mathrm{SD}=11.62)$ and seeing an average of $7.28(\mathrm{SD}=5.27)$ health care providers in the past year.

Of our 705 POTS participants, 213 (30\%) reported comorbid diagnosis of chronic fatigue syndrome/myalgic encephalomyelitis (CFS/ME), 237 (34\%) reported Ehlers-Danlos syndrome (EDS), and 85 (12\%) reported mast cell activation disorder (MCAD). A total of 29 (4\%) participants reported having the cluster of POTS, EDS, and $\mathrm{MCAD}^{33}$ as well as CFS/ME.

\section{Procedure}

Participants electronically signed the informed consent form before opening the online survey that included measures described here. Participants were given the option to complete the survey in one sitting or to return later. The study was approved by the Wittenberg University Institutional Review Board. All data were collected electronically via the PatientsCount.org website between April and June 2016.

\section{Self-reported questionnaires}

\section{Pittsburgh sleep quality index (PSQI)}

This nine-question self-report measure assesses sleep quality and dysfunction over the past month. ${ }^{31}$ It differentiates "poor" from "good" sleep quality by measuring seven areas on a $0-3$ point scale: subjective sleep quality, sleep latency, sleep duration, habitual sleep efficiency, sleep disturbances, use of sleeping medications, and daytime dysfunction over the last month. The total score ranges from $0-21$, with total scores over 5 indicating poor sleep quality.

\section{Suicide behaviors questionnaire-revised (SBQ-R)}

This four question self-report measure has been used to assign a designation of high or low suicide risk. ${ }^{32-36}$ The SBQ-R assesses lifetime suicidal ideation and/or suicide attempts. ${ }^{32}$ The SBQ-R questions are: (1) have you ever thought about or attempted to kill yourself? (score: $1-4 \mathrm{a} / \mathrm{b}$ ); (2) how often have you thought about killing yourself in the past year? (score: 1-5); (3) have you ever told someone that you were going to commit suicide, or that you might do it? (score: $1-3 \mathrm{a} / \mathrm{b}$ ); and (4) how likely is it that you will attempt suicide someday? (score: 0-6). The SBQ-R total score was used to group participants by suicidal risk. A score $\geq 7$ placed participants into the high suicidal risk group, while scores $<7$ placed participants in the low suicidal risk group. ${ }^{32,34}$

\section{Statistical analysis}

Statistical analyses were conducted using $\mathrm{R}$ computer software. Mean sleep scores and suicide scores for the POTS and control groups were compared with independent samples $t$-tests. Pearson correlations were used to measure relationships between suicidal ideation score and various aspects of sleep disturbance. Standard multiple linear regression was used to evaluate how well overall sleep quality, age, gender, and illness with POTS predicted suicidal ideation scores. 


\section{Results \\ Sleep}

According to the PSQI, 98.4\% of POTS patients surveyed had poor sleep quality (compared to $69.4 \%$ of controls), and the POTS group had significantly worse mean overall sleep scores than controls $(t[873]=17.75 ; p<0.001)$. The average POTS patient sleeps 6.0 hours per night which is significantly less than controls (Table 1, 7.8 hours; $t[873]=3.17 ; p<0.001$ ), despite being in bed for 9.45 hours. With a subjective sleep efficiency of $65.4 \%$, the POTS group had significantly less sleep efficiency than the control group $(t[873]=-11.32$; $p<0.001)$. The average sleep onset latency for those with POTS was 2 hours and 26 minutes.

A majority of POTS patients reported frequent (defined as happening $3+$ times per week) sleep disturbances from pain (53\%), failing to fall asleep within 30 minutes (59.9\%), and waking in the middle of the night or early morning (68.9\%; Table 2). Those with POTS had significantly more sleep disturbances because of pain than non-POTS controls $(t[873]=15.36 ; p<0.001)$. POTS patients also had significantly more disturbances from bad dreams $(t[873]=8.62 ; p<0.001)$, from breathing problems $(t[873]=11.13 ; p<0.001)$, from nighttime or early morning awakenings $(t[873]=7.70 ; p<0.001)$, and from failing to fall asleep within 30 minutes (Table 1; $t[873]=12.36 ; p<0.001)$. Further, POTS patients took sleeping medication significantly more frequently than non-POTS controls $(t[873])=7.09 ; p<0.001)$ and gave significantly lower self-ratings of sleep quality $(t[873]=12.91 ; p<0.001)$. While the PSQI rated $98.4 \%$ of POTS patients to have poor sleep, only $70.2 \%$ rated their own sleep as poor. There were no significant gender differences with regards to sleep except that among the control group, females had more frequent sleep disturbances from feeling too cold $(t[213]=3.16 ; p=0.002)$.

\section{Suicidal ideation}

Among the 705 POTS patients who completed the SBQ-R, there were no gender differences in any sub-scores or total score. Suicidal ideation scores were not significantly correlated with age. POTS patients had a mean total score of 7.0 , just at the cut-off for being considered at high risk $(\geq 7)$, and also significantly higher than controls' mean total score of $4.7(t[873]=8.0 ; p<0.001)$. Among POTS patients, 333 $(47.2 \%)$ were at high risk compared with 27 controls (15.9\%). Chi-square testing showed a larger proportion of high-risk individuals among POTS patients than among controls $\left(\chi^{2}\right.$ $[1, \mathrm{n}=875]=55.6 ; p<0.001)$.

POTS patients scored significantly higher than controls on each of the four SBQ-R sub-scales. POTS patients had significantly higher scores for lifetime suicide ideation $(\bar{x}=2.0, \mathrm{SD}=0.9)$ than controls $(\bar{x}=1.5, \mathrm{SD}=0.7 ; t[873]=6.78$; $p<0.001)$. POTS patients had a significantly higher suicide ideation frequency in the past 12 months $(\bar{x}=2.3, \mathrm{SD}=1.4)$ than controls $(\bar{x}=1.4, \mathrm{SD}=1.0 ; t[873]=7.97 ; p<0.001)$. Threat of suicide attempt was also significantly higher for POTS patients

Table 2 Percent of participants with particular sleep disturbances three or more times per week

\begin{tabular}{lll}
\hline Disturbance & POTS (\%) & Control (\%) \\
\hline Rate sleep quality as fairly or very bad & 70.2 & 25.8 \\
Wake in middle of night or early morning & 68.9 & 42.4 \\
Sleep onset latency >30 minutes & 59.9 & 15.3 \\
Have pain & 53.0 & 10.6 \\
Take medicine to help sleep & 40.9 & 16.5 \\
Cannot breathe comfortably & 23.8 & 3.5 \\
Have bad dreams & 18.3 & 1.8 \\
Considered to have overall poor sleep & 98.4 & 69.4 \\
per the PSQ & &
\end{tabular}

per the PSQI

Abbreviations: POTS, postural orthostatic tachycardia syndrome; PSQI, Pittsburgh Sleep Quality Index; SD, standard deviation.

Table I Sleep quality in POTS and controls as measured by PSQI

\begin{tabular}{|c|c|c|c|c|}
\hline Component of sleep quality & $\begin{array}{l}\text { POTS } \\
\text { Mean (SD) }\end{array}$ & $\begin{array}{l}\text { Control } \\
\text { Mean (SD) }\end{array}$ & $t(873)=$ & Significance \\
\hline Total sleep score (range 0-2I) & I3.3 (4.1) & $7.0(4.1)$ & 17.7 & $<0.001$ \\
\hline Time asleep (hours) & $6.0(2.4)$ & $7.8(2.0)$ & 5.8 & $<0.001$ \\
\hline Subjective sleep efficiency (\%) & $65.4(21.6)$ & $85.4(15.3)$ & -11.3 & $<0.001$ \\
\hline Frequency of sleep disturbances due to pain* & $2.2(I . I)$ & $0.8(1.0)$ & 15.4 & $<0.001$ \\
\hline Frequency of sleep disturbances due to bad dreams* & $\mathrm{I} .3(\mathrm{I} . \mathrm{I})$ & $0.6(0.7)$ & 8.6 & $<0.001$ \\
\hline Frequency of sleep disturbances due to breathing problems* & $\mathrm{I} .3(\mathrm{I} .2)$ & $0.3(0.7)$ & II.I & $<0.001$ \\
\hline Self-rated sleep quality* & $1.9(0.8)$ & I.I (0.7) & 12.9 & $<0.001$ \\
\hline Frequency of being unable to get to sleep within 30 minutes* & $2.2(1.1)$ & I.I (I.I) & 12.4 & $<0.001$ \\
\hline Frequency of waking in the middle of the night or early morning* & $2.5(0.9)$ & $1.9(1.1)$ & 7.7 & $<0.001$ \\
\hline Frequency of using sleep medicine* & $\mathrm{I} .5(\mathrm{I} .4)$ & $0.6(1.1)$ & 7.1 & $<0.001$ \\
\hline
\end{tabular}

Notes: *Single item on PSQI range 0-3. Higher scores indicate more sleep disturbance.

Abbreviations: POTS, postural orthostatic tachycardia syndrome; PSQI, Pittsburgh Sleep Quality Index; SD, standard deviation. 
$(\bar{x}=1.5, \mathrm{SD}=0.7)$ than controls $(\bar{x}=1.2, \mathrm{SD}=0.5 ; t[873]=5.50 ;$ $p<0.001)$. Those with POTS had a higher likelihood of suicidal behavior in the future $(\bar{x}=1.2 ; \mathrm{SD}=1.4)$ than controls $(\bar{x}=0.6$, $\mathrm{SD}=1.0 ; t[873]=5.35 ; p<0.001)$. As shown in Table $3,23.8 \%$ of POTS patients had made a suicide plan at some point (Item 1 , responses $3 \mathrm{a}$ and $3 \mathrm{~b}$ ), compared to $12.3 \%$ of controls, and $15.4 \%$ had made a suicide attempt (Item 1 , responses $4 \mathrm{a}$ and $4 \mathrm{~b}$ ), compared to $2.4 \%$ of controls. Among POTS patients, $12.3 \%$ reported "very frequent" suicide ideation in the past 12 months, compared to $1.2 \%$ of controls. Among POTS

Table 3 Responses to SBQ-R ${ }^{32}$

\begin{tabular}{lll}
\hline Response & $\begin{array}{l}\text { POTS } \\
\mathbf{n}(\%)\end{array}$ & $\begin{array}{l}\text { Controls } \\
\mathbf{n}(\%)\end{array}$ \\
\hline Item I: Lifetime suicide ideation/attempts: & $187(26.5)$ & $88(5 I .8)$ \\
$\begin{array}{l}\text { I. Never } \\
\text { 2. It was just a brief passing thought }\end{array}$ & $24 \mid(34.2)$ & $57(33.5)$ \\
$\begin{array}{l}\text { 3a. Had a plan at least once to kill myself but } \\
\text { did not try to do it }\end{array}$ & $94(13.3)$ & $12(7.0)$ \\
$\begin{array}{l}\text { 3b. Had a plan at least once to kill myself and } \\
\text { really wanted to die }\end{array}$ & $74(10.5)$ & $9(5.3)$ \\
$\begin{array}{l}\text { 4a. Attempted to kill myself, but did not want } \\
\text { to die }\end{array}$ & $4 I(5.8)$ & $3(1.8)$ \\
$\begin{array}{l}\text { 4b. Attempted to kill myself, and really hoped } \\
\text { to die }\end{array}$ & $68(9.6)$ & I (0.6)
\end{tabular}

Item 2: Suicide ideation frequency in the past 12 months:

I. Never

2. Rarely (I time)

3. Sometimes ( 2 times)

4. Often (3-4 times)

5. Very often (5 or more times)

Item 3: Threat of suicide attempt:

I. No

2a. Yes, one time, but did not really want to die

$2 b$. Yes, one time, and really wanted to die

3a. Yes, more than once, but did not want to do it

3b. Yes, more than once, and really wanted to do it

$\begin{array}{ll}309(43.8) & 134(78.8) \\ 134(19.0) & 19(11.2) \\ 111(15.7) & 9(5.3) \\ 64(9.1) & 6(3.5) \\ 87(12.3) & 2(1.2) \\ & \\ 448(63.5) & 141(82.9) \\ 87(12.3) & 21(12.4) \\ 65(9.2) & 5(2.9) \\ 46(6.5) & 2(1.2) \\ 59(8.4) & 1(0.6)\end{array}$

Item 4: Likelihood of suicidal behavior in the future:

0. Never

I. No chance at all

2. Rather unlikely

3. Unlikely

4. Likely

5. Rather likely

6. Very Likely

SBQ-R total score:

High risk

$\begin{array}{ll}315(44.6) & 110(64.7) \\ 127(18.0) & 27(15.9) \\ 142(20.2) & 25(14.7) \\ 70(9.9) & 6(3.5) \\ 33(4.7) & 2(1.2) \\ 9(1.3) & 0(0.0) \\ 9(1.3) & 0(0.0)\end{array}$

Note: Reprinted by permission of SAGE Publications, Inc. Osman A, Bagge CL, Gutierrez PM, Konick LC, Kopper BA, Barrios FX, Assessment. 2001;8(4):443-454, Copyright $\odot 2001$ by SAGE Publications. ${ }^{32}$

Abbreviations: POTS, postural orthostatic tachycardia syndrome; SBQ-R, Suicide Behaviors Questionnaire-Revised. patients, 36.5\% had threatened suicide (Item 3, responses $2 \mathrm{a}$, $2 \mathrm{~b}, 3 \mathrm{a}$, and $3 \mathrm{~b}$ ) compared to $17.1 \%$ of controls. When asked about likelihood of attempting suicide in the future, $7.3 \%$ of POTS patients said that they were likely to do so, compared to $1.2 \%$ of non-POTS controls (Table 3; Item 4, responses 4-6).

\section{Sleep and suicidal ideation}

Pearson correlations showed associations between suicidal ideation score and several components of the PSQI: subjective sleep efficiency $(r=-0.26, p<0.001)$; delayed sleep latency over 30 minutes $(r=0.22, p<0.001)$; having bad dreams $(r=0.29, p<0.001)$; disruption from pain $(r=0.24$, $p<0.001$ ); having trouble staying awake while driving, eating meals, or engaging in social activity $(r=0.29, p<0.001)$; keeping up enthusiasm to get things done $(r=0.36, p<0.001)$; self-rated overall sleep quality $(r=0.27, p<0.001)$; and overall sleep quality score $(r=0.36, p<0.001)$.

A significant linear regression model, $F(4,870)=38.34$, $p<0.001$, explained $15 \%$ of the variance in suicide ideation score $\left(R^{2}=0.15\right)$. Significant predictors were age $(\beta=-0.03$, $p=0.001)$, sleep quality as measured by total PSQI score $(\beta=0.23, p<0.001)$, and having POTS $(\beta=0.68, p=0.046)$. Gender was not a significant predictor $(\beta=0.44, p=0.46)$ of suicide ideation score.

\section{Discussion}

In our sample, online surveys revealed significantly more sleep disruption and suicidal ideology in the POTS group than in the control group. More than $50 \%$ of our POTS participants reported poor sleep, nighttime wakefulness, sleep onset latency $>30$ minutes, and sleep disruption due to pain. Further, people with POTS took more medications to help them sleep than controls. Those in the POTS group were also more likely to consider suicide. Particularly associated with suicidal ideation among those with POTS were increased frequency of bad dreams, pain that disrupted sleep, poor subjective sleep efficiency, sleep onset latency $>30$ minutes, difficulty staying awake during the day, self-ratings of worse sleep quality, and keeping up enthusiasm to get things done. Significant predictors of suicidal ideation in this study were sleep quality, having POTS, and age. Overall, the POTS group had a significantly higher risk for suicidal ideation as well as past and future attempts than the control group.

Sleep disturbances, including non-restorative sleep and insomnia, are a common complaint for many with POTS who tend to have poor sleep, high rates of fatigue, and decreased quality of life. ${ }^{4,10,12,37}$ Poor sleep efficiency has been demonstrated in other studies of POTS patients. ${ }^{5,11,12}$ In this 
study, more than $50 \%$ of POTS patients reported poor sleep, nighttime wakefulness, sleep onset latency $>30$ minutes, and pain disrupting their sleep. This is higher than other studies in which nearly $30-40 \%$ of POTS patients complained of sleep disturbance or poor sleep quality. ${ }^{1,11}$

Not all studies have found gross sleep disturbance in POTS patients. Using polysomnography, POTS patients demonstrated normal total sleep time, no significant sleep fragmentation, and no significant sleep disturbance despite subjective reports of poor sleep. ${ }^{11,15}$ Despite these appearances of normal sleep, Pengo et al found a reduction in REM sleep, ${ }^{15}$ while Mallien et al reported a diminished heart rate variability in those with POTS during various sleep stages. ${ }^{11}$ Perhaps the subjective feeling of poor sleep quality in this and other studies is linked to these poorly understood physiological differences during sleep in people with POTS.

Approximately $50 \%$ of people with invisible illness also suffer from depression. ${ }^{20}$ The mechanisms underlying depression in POTS patients have not been well studied. Moon et al found that severity of concentration difficulties and chest discomfort were most strongly correlated with depression in POTS patients. ${ }^{38}$ Another small study $(n=15)$ found that the majority of POTS patients suffered from depression, ${ }^{2}$ while Raj et al found that the lifetime prevalence of major depressive disorder was not elevated. ${ }^{39}$ Clearly, the relationship between POTS and depression and their effect on disturbed sleep will require more study.

Sleep disturbances, including insomnia and nightmares, can increase suicidal ideation, ${ }^{16,18,40-43}$ perhaps by increasing depression and eventually suicidal ideation. ${ }^{17,19}$ Interestingly, some studies have shown that sleep disturbances can increase suicidal ideation and behavior independent of depression. ${ }^{21,22}$ Persistent nightmares have also been linked to increased suicidal ideology independent of insomnia. ${ }^{19,23}$ In this study, poor subjective sleep efficiency, increased frequency of delayed sleep latency, bad dreams, difficulty staying awake during the day, and pain disrupting sleep were correlated with increased suicidal ideation. Poor sleep has been estimated to account for $50 \%$ of quality of life issues in people with POTS,${ }^{10}$ and is clearly a health issue that needs to be more adequately addressed.

There are a number of possible mediators for the number of POTS patients (47.2\%) in the high risk group for suicide. Age can play a factor in suicide risk. Our POTS group was composed of predominately young women (mean age 33.75 years). At this age (25-44 years), women tend to die by suicide at a much lower rate than men, and women between the ages of 45 and 64 years. ${ }^{44}$ Sleep medications could also affect both sleep architecture and quality that might increase suicide rates in this population. Sedatives and hypnotic medications, for example, have been shown to increase suicide risk and can be a stronger predictor than insomnia for suicidal ideation and behavior. ${ }^{45,46}$ Future studies should account for prescribed sleep medications.

\section{Limitations}

There are limitations to our study. All data were taken from online self-report measures, rather than objective physical assessment data or direct physician diagnosis. Participants reported having physician diagnosed POTS, and some had been diagnosed with CFS, EDS, and MCAD. Some may have had other comorbid diagnoses that could worsen their symptomology and increase suicidal ideology. Because self-reports of sleep were used in this study, it is difficult to uncover the mechanisms that link sleep disruption with suicidal tendencies in this population. Perhaps depression was a mediator in the suicidal ideation and behavior, but that data was not collected in this study. In addition, POTS participants were likely using both pharmaceutical and non-pharmaceutical treatments, and we have no control for sleep medications and other aspects to their treatment regimen. The use of sleep medications could influence sleep architecture and perhaps indirectly influence the self-report measures. Finally, because our sample was partially solicited from online support groups, our sample may have been biased toward those with more debilitating POTS symptoms which motivate them to seek advice and support in these forums.

\section{Conclusion}

Sleep disturbance is an important factor in quality of life for many chronically ill people, including those with POTS. The relative lack of good data on the mechanistic links between POTS, depression, and disturbed sleep might have limited the variability explained in the current model. Sleep issues can be properly treated through use of pharmacologic and nonpharmacologic means. ${ }^{47,48}$ In POTS, treatments for insomnia may include medications to decrease sympathetic activation like clonidine, and non-pharmacological means like improving sleep hygiene, sleep restriction, mild exercise. ${ }^{47,49,50}$ It is important, therefore, that health care providers prioritize issues of poor sleep quality when working with POTS patients to decrease suicide risk.

\section{Disclosure}

The authors report no conflicts of interest in this work. 


\section{References}

1. Thieben MJ, Sandroni P, Sletten DM, et al. Postural orthostatic tachycardia syndrome: the Mayo clinic experience. Mayo Clinic Proc. 2007; 82(3):308-313.

2. Anderson JW, Lambert EA, Sari CI, et al. Cognitive function, healthrelated quality of life, and symptoms of depression and anxiety sensitivity are impaired in patients with the postural orthostatic tachycardia syndrome (POTS). Front Physiol. 2014;5:1-9.

3. Benrud-Larson LM, Dewar MS, Sandroni P, Rummans TA, Haythornthwaite JA, Low PA. Quality of life in patients with postural tachycardia syndrome. Mayo Clinic Proc. 2002;77:531-537.

4. Benarroch EE. Postural tachycardia syndrome: a heterogeneous and multifactorial disorder. Mayo Clinic Proc. 2012;87(12):1214-1225.

5. Bagai K, Wakwe CI, Malow B, et al. Estimation of sleep disturbances using wrist actigraphy in patients with postural tachycardia syndrome. Auton Neurosci. 2013;177:260-265.

6. Deb A, Morgenshtern K, Culbertson CJ, Wang LB, DePold Hohler A. A survey-based analysis of symptoms in patients with postural orthostatic tachycardia syndrome. Proc Bayl Univ Medl Cent. 2015;28(2):157-159.

7. Braune S, Wrockiage C, Schulte-Monting J, Schnitzer R, Lucking CH. Diagnosis of tachycardia syndromes associated with orthostatic symptoms. Clin Auton Res. 1999;9(2):97-101.

8. Raj SR. Highlights in clinical autonomic neurosciences: treatment insights for postural tachycardia syndrome and inappropriate sinus tachycardia. Auton Neurosci. 2013;177(2):72-73.

9. Grubb BP, Karabin B. Postural tachycardia syndrome: perspectives for patients. Circulation. 2008;118:e61-e62.

10. Bagai K, Song Y, Ling JF, et al. Sleep disturbance and diminished quality of life in postural tachycardia syndrome. J Clin Sleep Med. 2011; 7(2):204-210.

11. Mallien J, Isenmann S, Mrazek A, Haensch C-A. Sleep disturbances and autonomic dysfunction in patients with postural orthostatic tachycardia syndrome. Front Neurol. 2014;5:118-124.

12. Xu X, Huang H, Sethi S, Zuzuarregui JRP, Weinberg J, Hohler AD. A survey based study on sleep disturbance in postural tachycardia syndrome. J Neurolog Sci. 2016;365:199-202.

13. Bagai K, Peltier AC, Malow BA, et al. Objective Sleep Assessments in Patients with Postural Tachycardia Syndrome using Overnight Polysomnograms. J Clin Sleep Med. 2016;12(5):727-733.

14. Miglis MG, Muppidi S, Feakins C, Fong L, Prieto T, Jaradeh S. Sleep disorders in patients with postural tachycardia syndrome. Clin Auton Res. 2016;26(1):67-73.

15. Pengo MF, Higgins S, Drakatos $\mathrm{P}$, et al. Characterisation of sleep disturbances in postural orthostatic tachycardia syndrome: a polysomnography-based study. Sleep Med. 2015;16:1457-1461.

16. Bernert R, Kim JS, Iwata NG, Perlis ML. Sleep disturbances as an evidence-based suicide risk factor. Curr Psychiatry Rep. 2015;17(3): $15-24$.

17. Bozzay ML, Karver MS, Verona E. Linking insomnia and suicide ideation in college females: the role of socio-cognitive variables and depressive symptoms in suicide risk. J Affect Disord. 2016;199(1-C): 106-113.

18. McCall WV, Black CG. The link between suicide and insomnia: theoretical mechanisms. Curr Psychiatry Rep. 2013;15(9):389-398.

19. Cukrowicz KC, OtamendiA, Pinto JV, Bernet RA, Krakow B, Joiner Jr. TE. The impact of insomnia and sleep disturbances on depression and suicidality. Dreaming. 2006;16(1):1-10.

20. Nicholas M. Depression in people with pain: there is still work to do. Scandinavian Journal of Pain. 2011;2(2):45-46.

21. Pigeon WR, Pinquart M, Conner K. Meta-analysis of sleep disturbance and suicidal thoughts and behaviors. J Clin Psychiatry. 2012; 73(9):e1160-e1167.

22. Ribeiro JD, Pease JL, Gutierrez PM, et al. Sleep problems outperform depression and hopelessness as cross-sectional and longitudinal predictors of suicidal ideation and behavior in young adults in the military. J Affect Disord. 2012;136(3):743-750.
23. Sjostrom N, Hetta J, Waern M. Persistent nightmares are associated with repeat suicide attempt. Psychiatry Res. 2009;170(2-3):208-211.

24. Mann JJ, Apter A, Bertolote J, et al. Suicide prevention strategies: a systematic review. JAMA. 2005;294:2064-2074.

25. Jason LA, Corradi K, Gress S, Williams S, Torres-Harding S. Causes of death among patients with chronic fatigue syndrome. Health Care Women Int. 2006;27:615-626.

26. Saez-Francas N, Calvo N, Alegre J, et al. Childhood trauma in chronic fatigue syndrome: focus on personality disorders and psychopathology. Compr Psychiatry. 2015;62:13-19.

27. Calandre EP, Vilchez JS, Molina-Barea R, et al. Suicide attempts and risk of suicide in patients with fibromyalgia: a survey in Spanish patients. Rheumatology. 2011;50(10):1889-1893.

28. Dreyer L, Kendall S, Danneskiold-Samsoe B, Bartels EM, Bliddal H. Mortality in a cohort of Danish patients with fibromyalgia: increased frequency of suicide. Arthritis Rheum. 2010;62(10):3101-3108.

29. Trinanes Y, Gonzalez-VillarA, Gomez-Perretta C, Carrillo-de-la-Pena MT. Suicidality in chronic pain: predictors of suicidal ideation in fibromyalgia. Pain Practice. 2015;15(4):323-332.

30. Wolfe F, Hassett AL, Walitt B, Michaud K. Mortality in fibromyalgia: a study of 8,186 patients over thirty-five years. Arthritis Care Res (Hoboken). 2011;63(1):94-101.

31. Buysse DJ, Reynolds CF, Monk TH, Berman SR, Kupfer DJ. The Pittsburgh sleep quality index: a new instrument for psychiatric practice and research. Psychiatry Res. 1989;28:193-213.

32. Osman A, Bagge CL, Gutierrez PM, Konick LC, Kopper BA, Barrios FX. The suicidal behaviors questionnaire-revised (SBQ-R): validation with clinical and nonclinical samples. Assessment. 2001;8(4):443-454.

33. Cheung I, Vadas P. A new disease cluster: mast cell activation syndrome, postural orthostatic tachycardia syndrome, and Ehlers-Danlos syndrome. J Allergy Clin Immunol. 2015;135(2):AB65.

34. Farabaugh A, Nyer M, Holt D, et al. Screening for suicide risk in the college population. Journal of Rational-Emotive \& Cognitive-Behavior Therapy. 2015;33(1):78-94.

35. Koweszko T, Gierus J, Mosiolek A, Kaminski M, Wisniewska K, Szulc A. Differences in assessment of suicidal tendencies in men and women: a pilot study. Arch Psychiatr Nurs. 2016;30(1):77-78.

36. Wagner B, Klinitzke G, Brahler E, Kersting A. Extreme obesity is associated with suicidal behavior and suicide attempts in adults: results of a population-based representative sample. Depress Anxiety. 2013;30(10):975-981.

37. Ojha A, Chelimsky TC, Chelimsky G. Comorbidities in pediatric patients with postural orthostatic tachycardia syndrome. J Pediatr. 2011;158: 119-122.

38. Moon J, Kim DY, Byun JI, et al. Orthostatic intolerance symptoms are associated with depression and diminished quality of life in patients with postural tachycardia syndrome. Health Qual Life Outcomes. 2016; 14(1):144

39. Raj V, Haman KL, Raj SR, et al. Psychiatric profile and attention deficits in postural tachycardia syndrome. J Neurol Neurosurg Psychiatry. 2009;80(3):339-344.

40. Chellappa SL, Araujo JF. Sleep disorders and suicidal ideation in patients with depressive disorder. Psychiatry Res. 2007;153(2):131-136.

41. Woznica AA, Carney CE, Kuo JR, Moss TG. The insomnia and suicide link: toward an enhanced understanding of this relationship. Sleep Med Rev. 2015;22:37-46.

42. Nadorff MR, Anestis MD, Nazem S, Harris HC, Winer ES. Sleep disorders and the interpersonal-psychological theory of suicide: independent pathways to suicidality. J Affect Disord. 2014;152-154:505-512.

43. Wong MM, Brower KJ, Zucker RA. Sleep problems, suicidal ideation, and self-harm behaviors in adolescence. J Psychiatric Res. 2011;45(4): 505-511.

44. Curtin SC, Warner M, Hedegaard H. Increase in suicide in the United States, 1999-2014. 2016; https:/www.cdc.gov/nchs/products/databriefs/db241.htm. Accessed December 24, 2016.

45. Carlsten A, Waern M. Are sedatives and hypnotics associated with increased suicide risk of suicide in the elderly? BMC Geriatrics. 2009;9(20) 
46. Brower KJ, McCammon RJ, Wojnar M, Ilgen MA, Wojnar J, Valenstein M. Prescription sleeping pills, insomnia, and suicidality in the National Comorbidity Survey replication. J Clin Psychiatry. 2011;72(4):515-521.

47. Lynch AM, Jarvis CI, DeBellis RJ, Morin AK. Nonpharmacologic approaches for the treatment of insomnia. Am J Lifestyle Med. 2007;1(4): 274-282.
48. Thanavaro JL, Thanavaro KL. Postural orthostatic tachycardia syndrome: diagnosis and treatment. Heart Lung. 2011;40(6):554-560.

49. Conner R, Sheikh M, Grubb B. Postural orthostatic tachycardia syndrome (POTS): evaluation and management. Br J Med Pract. 2012;5(4):540.

50. Sidhu B, Obiechina N, Rattu N, Mitra S. Postural orthostatic tachycardia syndrome (POTS). BMJ Case Rep. 2013.
Nature and Science of Sleep is an international, peer-reviewed, open access journal covering all aspects of sleep science and sleep medicine, including the neurophysiology and functions of sleep, the genetics of sleep, sleep and society, biological rhythms, dreaming, sleep disorders and therapy, and strategies to optimize healthy sleep. The manuscript

\section{Dovepress}

management system is completely online and includes a very quick and fair peer-review system, which is all easy to use. Visit http://www. dovepress.com/testimonials.php to read real quotes from published authors.

Submit your manuscript here: https://www.dovepress.com/nature-and-science-of-sleep-journal 